\title{
Linkage disequilibrium score regression distinguishes associations between lean body mass and blood plasma proteome
}

Lu Zhang ( $18829532956 @ 163 . c o m$ )

Xi'an Jiaotong University https://orcid.org/0000-0003-4674-0250

Yan Zhao

Xi'an Jiaotong University

Yan Wen

Xi'an Jiaotong University

Mei Ma

Xi'an Jiaotong University

Shiqiang Cheng

Xi'an Jiaotong University

Bolun Cheng

Xi'an Jiaotong University

Li Liu

Xi'an Jiaotong University

Ping Li

Xi'an Jiaotong University

Xin Qi

Xi'an Jiaotong University

Chujun Liang

Xi'an Jiaotong University

Feng Zhang

Xi'an Jiaotong University

\section{Research article}

Keywords: genetic; genome-wide association study (GWAS); lean body mass; linkage disequilibrium score regression; plasma proteins

Posted Date: October 1st, 2019

DOI: https://doi.org/10.21203/rs.2.15375/v1 
License: (c) (i) This work is licensed under a Creative Commons Attribution 4.0 International License. Read Full License 


\section{Abstract}

Background: Lean body mass (LBM) is an essential component of body composition. Recent studies observed associations between altered plasma proteins and LBM. This study aims to explore the potential genetic relationships between LBM and plasma proteome.

Methods: The genome-wide association study (GWAS) summary data of LBM were driven from 85,000 individuals of European descent. The GWAS summary data of 3,622 plasma proteins were obtained from 3,301 healthy participants. Linkage disequilibrium score regression (LDSC) analysis were conducted to evaluate the potential genetic correlation between each plasma protein and LBM using the GWAS summary statistics.

Results: LDSC scan identified multiple plasma proteins for LBM, such as stabilin-1(coefficient=-0.4583, $P$ value $=0.0253$ ) and inter-alpha-trypsin inhibitor heavy chain $\mathrm{H} 1$ (coefficient $=-0.1716, P$ value $=0.0314$ ) for append LBM, and glyceraldehyde-3-phosphate dehydrogenase (coefficient $=0.5997, P$ value $=0.0315$ ) and immunoglobulin lambda-like polypeptide 1 (coefficient $=0.35, P$ value $=0.0334$ ) for whole LBM. We also detected two common plasma proteins shared by append and whole LBM, including stabilin-1 and glyceraldehyde-3-phosphate dehydrogenase.

Conclusions: This study provided an atlas of genetic correlations between LBM and plasma proteome, which are helpful for understanding the biological mechanism of LBM.

\section{Introduction}

Lean body mass (LBM) is a component of body composition. It is calculated by subtracting body fat weight from total body weight. Decreases in LBM can cause sarcopenia, which is characterized by absolute or relative reduction of muscle mass [1,2]. Loss of muscle mass results in decreased muscle strength, and impacts functional capacity/health negatively[3, 4], for example, osteoporosis, fracture, obesity, insulin resistance, overall frailty and increased mortality. Epidemiological research has shown that sarcopenia is present in about $5 \%$ to $10 \%$ of persons over 65 years, causing significant functional impairment [5].

Multiple factors contribute to the variation of LBM, such as exercise and protein intake [6]. Genetic factor also plays essential roles in the development of LBM. For instance, Lenchik et al. showed that LBM was a highly heritable trait with estimated heritability of $0.52-0.60$ [7]. Furthermore, multiple LBM associated genes have been identified, such as TRHR, DARC and MTHFR [8-10]. However, the heritability of LBM explained by the identified loci is limited. The genetic basis of LBM remains elusive now.

Plasma proteome are a group of proteins in plasma which are functionally implicated in signaling, transport, repair, and defense against infection. Human blood proteome provides a foundation for biomarkers identification, disease diagnosis and development of new therapeutic products [11]. It has been found that altered plasma proteins were related to multiple human complex traits and diseases, 
including sarcopenia. For instance, plasma transthyretin (TTR) is closely superimposable to LBM, thus TTR works as the best surrogate analyte of LBM [12]. Kishiko et al. found that eHsp72 in plasma was closely related to sarcopenia, and it was a potential biomarker or predictor for sarcopenia [13]. However, limited efforts are paid to systematically explore the relationships between LBM and plasma proteome.

It is well known that differences in protein function or abundance are in most cases responsible for phenotypic differences among different diseases and susceptibility to diseases. To date, gene expression quantitative trait loci (eQTL) mapping has successfully provided insights into the underlying molecular mechanisms of complex diseases [14]. However, this approach explains only $40 \%$ of the variability in protein levels [15]. Therefore, using the proteome, which might be more closely related to the phenotype level, as a surrogate, may acquire additionally useful information for the pathogenetic studies of complex diseases. Holdt et al. recently performed a proteomic quantitative trait locus (pQTL) study of plasma proteome screens. They observed that genome-wide plasma proteome profiling in combination with genome-wide genetic screening aided in the identification of causal genetic variants affecting abundance of plasma proteins[16].

Recent studies demonstrated the generality of genetic correlations among complex traits and diseases [17]. Genetic correlation is a key population parameter that describes the shared genetic architectures among different complex traits and diseases. It can be estimated by an efficient approach, named linkage disequilibrium score regression (LDSC)[18]. LDSC is capable of quantifying the genetic relationships among various diseases and traits, utilizing genome-wide association study (GWAS) summary data and linkage disequilibrium (LD) information. Utilizing LDSC, Bulik-Sullivan B et al. evaluated the 276 genetic relationships among 24 traits, and observed significant genetic correlations between anorexia nervosa and schizophrenia, anorexia and obesity [19].

In this study, we conducted a large-scale LDSC scan to detect the potential genetic correlations between human plasma proteome and LBM. The latest GWAS summary data of 3,622 plasma proteins and LBM were used here. Our results provide novel clues for understanding the genetic mechanism underlying the variation of LBM.

\section{Materials And Methods}

\section{GWAS summary data of LBM}

A recent large-scale GWAS dataset of LBM was used here [20]. Briefly, this GWAS contained $\sim 85,000$ individuals of European descent from 47 studies. Whole body lean mass and appendicular lean mass were measured using either DXA or BIA. Genotype imputation of all studies was performed by IMPUTE or $\mathrm{MACH}$ software using the HapMap Phase II release 22 reference panel. Phenotype-genotype association was tested by multiple linear regression model with additive genetic effect, using 2.0 to 2.5 million genotyped and/or imputed autosomal SNPs. Finally, meta-analysis was conducted using an inverse variance weighting and fixed-effect model approach. Detailed description of study participantss, 
genotyping, imputation, association analysis and quality control could be found in the published study [20].

\section{GWAS data of human plasma proteome}

The GWAS data of human plasma proteome was derived from a recently published study [21]. In brief, a multiplexed, aptamer-based approach (SOMAscan assay) was used to measure the relative concentrations of 3,622 plasma proteins in 3,301 healthy participants[21]. Genotyping was performed using Affymetrix Axiom UK Biobank genotyping array. Genotype imputation was performed by Sanger Imputation Server (https://imputation.sanger.ac.uk). Association analysis was performed by linear regression using an additive genetic model. Detailed description of participants, genotyping, imputation, association analysis and quality control could be found in the published study[21].

\section{Genetic correlation scanning}

Follow the approach recommended by the developers, LDSC software (v1.0.0; https://github.com/bulik/ldsc) was applied to the GWAS summary data of LBM and plasma proteome for evaluating the genetic correlations between LBM and each plasma protein. The basic principle of the LDSC approach is to estimate directly from GWAS summary data using the deviation of the observed $\chi^{2}$ test statistic for a Single Nucleotide Polymorphisms(SNP) from its expected value under the null hypothesis of no association[22]. This method is very flexible and can be adapted to estimate the genetic correlation among different complex traits and diseases[23].

\section{Results}

LDSC analysis identified 4 plasma proteins with LDSC $\mathrm{P}$ values $<0.05$ for append lean mass (Figure 1), including stabilin -1 (coefficient $=-0.4583, \mathrm{P}$ value $=0.0253$ ), inter-alpha-trypsin inhibitor heavy chain $\mathrm{H} 1$ (coefficient $=-0.1716, \mathrm{P}$ value $=0.0314$ ) and peregrin (coefficient $=-0.4602, \mathrm{P}$ value $=0.0353$ ) and glyceraldehyde-3-phosphate dehydrogenase (coefficient $=0.6043, \mathrm{P}$ value $=0.0325$ ) .

For whole body lean mass, we detected 4 plasma proteins with LDSC $P$ values $<0.05$, including stabilin1 (coefficient $=-0.4562, \mathrm{P}$ value $=0.0223)$, glyceraldehyde -3 -phosphate dehydrogenase $($ coefficient $=$ $0.5997, \mathrm{P}$ value $=0.0315$ ), immunoglobulin lambda-like polypeptide 1 (coefficient $=0.35, \mathrm{P}$ value $=$ 0.0334 ) and growth/differentiation factor 8 (coefficient $=0.3842, \mathrm{P}$ value $=0.0481$ ). Interestingly, we found that stabilin-1 and glyceraldehyde-3-phosphate dehydrogenase were detected for both append lean mass and whole body lean mass in this study. Detailed LDSC analysis results of LBM are summarized in Table 1 and Figure 1.

\section{Discussion}

The cellular and molecular basis of LBM is a field of enormous interest over the past few years. However, the biological mechanism of LBM is complex and remains largely unknown. Recent studies 
demonstrated that genetic factor contributed greatly to the development of LBM [7]. Additionally, altered plasma proteins have been found to be related to sarcopenia[12]. However, few efforts were paid to systematically explore the relationships between LBM and plasma proteome. In this study, we conducted a large scale LDSC analysis to explore the genetic correlations between LBM and human plasma proteome. We detected multiple candidate plasma proteins showing genetic correlation evidence with LBM. We hope our results provide novel clues for understanding the molecular mechanism of LBM.

We detected 8 plasma proteins showing genetic correlation evidence with LBM, such as myostatin and glyceraldehyde-3-phosphate dehydrogenase (GAPDH). Myostatin (growth/differentiation factor-8, GDF-8) belongs to the transforming growth factor $b$ super family of secreted proteins, controlling growth and differentiation. Myostatin expresses uniquely in human skeletal muscle and secrets into the plasma $[24,25]$. Zhiliang et al. reported that myostatin in chickens played an important role in controlling skeletal muscle growth and differentiation [26]. Another study showed that deletion and loss of function mutations in myostatin caused skeletal muscle hyperplasia and hypertrophy [27]. These cellular adaptations yield a hypermuscular phenotype in multiple species, including humans [27]. Additionally, in transgenic animal models, a lack of myostatin appeared to reduce age-related sarcopenia and loss of muscle regenerative capacity[28]. Current research indicated that myostatin inhibition could be used as a strategy to counter sarcopenia [29]. Furthermore, it was suggested that exercise in the form of endurance or resistance training would suppress muscle myostatin expression and reverse or limit the development of sarcopenia [30].

GAPDH is an enzyme of the glycolytic pathway that catalyzes the oxidation and phosphorylation of glyceraldehyde-3-phosphate by NAD+ and Pi[31]. It has been demonstrated that GAPDH is a major constituent of the intact muscle[32]. Additionally, a previous study has shown that modification of the kinetic properties of GAPDH with solubilization or binding was important in the regulatory mechanisms of the muscle cell[33]. Robert et al. measured the expression of GAPDH mRNA in a panel of 72 different pathologically normal human tissue types, and observed the highest express level in skeletal muscle[34]. Furthermore, Dawn A et al found that the lower glycolytic capacity of muscles from aged animals may be related to reduced transcription because GAPDH mRNA levels were lower in many muscles of the senescent rats[35]. It has been shown that denervation, chronic stimulation, and overload-induced hypertrophy caused GAPDH mRNA levels in skeletal muscles to decline [36-39].

Extensive efforts have been paid to explore the genetic mechanism of gene expression regulation, and identified a lot of eQTLs. However, most methods can only explain part of the variation of protein levels. In this study, we used latest GWAS data of blood proteins and LBM to systematically evaluate the genetic relationships between LBM and human plasma proteome by LDSC approach. Using the proteome as a surrogate, our approach can be seen as a supplement which might be more closely related to the phenotype level.

Two limitations should be noted in our study. First, the objective of this study is to evaluate the genetic correlations between plasma proteome and LBM, and to scan novel candidate plasma proteins related to 
LBM. Therefore, further functional studies are needed to confirm our findings and clarify the potential biological mechanisms of observed associations between plasma proteins and LBM in this study. Second, plasma protein levels may not reflect the protein levels within tissues or cells.

In summary, we conducted a large-scale analysis to investigate the genetic correlations between blood plasma proteome and LBM utilizing LDSC approach. Our study identified a set of candidate plasma proteins showing association signals with LBM. Nevertheless, more research on the investigated plasma proteins needs to be done to draw comprehensive conclusions about a plasma proteins and its association with LBM. We hope that our findings will provide novel insights into future pathogenetic studies of sarcopenia. Our results may also serve as a fundamental resource for understanding the genetic mechanisms of the effects of plasma proteome on LBM.

\section{List Of Abbreviations}

Lean body mass (LBM)

Genome wide association study (GWAS)

Linkage disequilibrium score regression (LDSC)

Transthyretin (TTR)

Expression quantitative trait loci (eQTL)

Proteomic quantitative trait locus ( $\mathrm{pQTL}$ )

Linkage disequilibrium

Single Nucleotide Polymorphisms(SNP)

Glyceraldehyde-3-phosphate dehydrogenase (GAPDH)

\section{Declarations}

\section{Ethics approval and consent to participate}

Not applicable

\section{Consent for publication}

Not applicable 


\section{Availability of data and materials}

Not applicable

\section{Competing interests}

The authors declare that they have no competing interests.

\section{Funding}

This work was supported by the National Natural Scientific Foundation of China (81673112); and the Key projects of international cooperation among governments in scientific and technological innovation [2016YFE0119100].

\section{Authors' contributions}

We would like to thank Zhang and Zhao drafted the manuscript, Zhang designed the study. Ma, Cheng, Liu and Li performed the statistical analyses. Wen, Cheng, Liang and Qi provided feasible advice on data analysis and drafting manuscript.

\section{Acknowledgements}

Not applicable

\section{References}

[1]R. Roubenoff, Sarcopenia: a major modifiable cause of frailty in the elderly Journal of Nutrition Health \& Aging 4 (2000) 140-142.

[2]J. E. Morley, R. N. Baumgartner, R. Roubenoff, J. Mayer, K. S. Nair, Sarcopenia Journal of Laboratory \& Clinical Medicine 137 (2001) 231-243.

[3]L. Fulvio, R. Cosimo Roberto, B. Stefania, B. Benedetta, C. Chiara, D. I. Angelo, C. Anna Maria, R. Taina, J. M. Guralnik, F. Luigi, Age-associated changes in skeletal muscles and their effect on mobility: an operational diagnosis of sarcopenia Journal of Applied Physiology 95 (2003) 1851-1860.

[4]I. Janssen, S. B. Heymsfield, R. Ross, Low Relative Skeletal Muscle Mass (Sarcopenia) in Older Persons Is Associated with Functional Impairment and Physical Disability Journal of the American Geriatrics Society 50 (2010) 889-896. 
[5]J. E. Morley, S. D. Anker, S. V. Haehling, Prevalence, incidence, and clinical impact of sarcopenia: facts, numbers, and epidemiology-update 2014 J Cachexia Sarcopenia Muscle 5 (2014) 253-259.

[6]D. K. Houston, B. J. Nicklas, J. Ding, T. B. Harris, F. A. Tylavsky, A. B. Newman, J. S. Lee, N. R. Sahyoun, M. Visser, S. B. Kritchevsky, Dietary protein intake is associated with lean mass change in older, community-dwelling adults: the Health, Aging, and Body Composition (Health ABC) Study American Journal of Clinical Nutrition 87 (2008) 150-155.

[7]F. C. Hsu, L. Lenchik, B. J. Nicklas, K. Lohman, T. C. Register, J. Mychaleckyj, C. D. Langefeld, B. I. Freedman, D. W. Bowden, J. J. Carr, Heritability of body composition measured by DXA in the diabetes heart study Obesity research 13 (2012) 312-319.

[8]X. G. Liu, L. J. Tan, S. F. Lei, Y. J. Liu, H. Shen, L. Wang, H. Yan, Y. F. Guo, D. H. Xiong, X. D. Chen, Genome-wide association and replication studies identified TRHR as an important gene for lean body mass American Journal of Human Genetics 84 (2009) 418-423.

[9]R. Hai, L. Zhang, Y. F. Pei, L. J. Zhao, S. Ran, Y. Y. Han, X. Z. Zhu, H. Shen, Q. Tian, H. W. Deng, Bivariate genome-wide association study suggests that the DARC gene influences lean body mass and age at menarche Science China 55 (2012) 516-520.

[10]X. Liu, L. J. Zhao, Y. J. Liu, D. H. Xiong, R. R. Recker, H. W. Deng, The MTHFR gene polymorphism is associated with lean body mass but not fat body mass Human Genetics 123 (2008) 189-196.

[11]B. B. Sun, J. C. Maranville, J. E. Peters, S. David, J. R. Staley, B. James, B. Stephen, J. Tao, P. Ellie, S. Praveen, Genomic atlas of the human plasma proteome Nature.

[12]I. Yves, L. H. Bernstein, Plasma Transthyretin as a Biomarker of Lean Body Mass and Catabolic States Advances in Nutrition 6 (2015) 572-580.

[13]O. Kishiko, K. Hun-Kyung, S. Takahiko, A. Sigeaki, S. Yumi, S. K. Calderwood, Plasma heat shock protein 72 as a biomarker of sarcopenia in elderly people Cell Stress \& Chaperones 17 (2012) 349-359.

[14]C. William, L. Liming, A. Gon?Alo, M. Miriam, L. Mark, Mapping complex disease traits with global gene expression Nature Reviews Genetics 10 (2009) 184-194.

[15]S. U. Bj?Rn, B. Dorothea, L. Na, D. Gunnar, S. Johannes, W. Jana, C. Wei, S. Matthias, Corrigendum: Global quantification of mammalian gene expression control Nature 495 (2013) 126-127.

[16]L. M. Holdt, V. D. Annette, N. Alexandros, B. Sven, K. Markus, T. Joachim, T. Daniel, Quantitative trait loci mapping of the mouse plasma proteome (pQTL) Genetics 193 (2013) 601.

[17]E. L. Grigorenko, Understanding the Etiology of Complex Traits: Symbiotic Relationships Between Psychology and Genetics Mind Brain \& Education 1 (2010) 193-199. 
[18]S. Ripke, B. M. Neale, A. Corvin, J. T. R. Walters, K. H. Farh, P. A. Holmans, P. Lee, B. Bulik-Sullivan, D. A. Collier, H. Huang, Estimation of Genetic Correlation via Linkage Disequilibrium Score Regression and Genomic Restricted Maximum Likelihood American Journal of Human Genetics 102 (2018).

[19]B. S. Brendan, H. K. Finucane, A. Verneri, G. Alexander, F. R. Day, L. Po-Ru, D. Laramie, J. R. B. Perry, P. Nick, E. B. Robinson, An atlas of genetic correlations across human diseases and traits Nature Genetics 47 (2015) 1236-1241.

[20]M. C. Zillikens, S. Demissie, Y. H. Hsu, L. M. Yergesarmstrong, W. C. Chou, L. Stolk, G. Livshits, L. Broer, T. Johnson, D. L. Koller, Large meta-analysis of genome-wide association studies identifies five loci for lean body mass Nature Communications 8 (2017) 80.

[21]B. B. Sun, J. C. Maranville, J. E. Peters, D. Stacey, J. R. Staley, J. Blackshaw, S. Burgess, T. Jiang, E. Paige, P. Surendran, C. Oliver-Williams, M. A. Kamat, B. P. Prins, S. K. Wilcox, E. S. Zimmerman, A. Chi, N. Bansal, S. L. Spain, A.M. Wood, N. W. Morrell, J. R. Bradley, N. Janjic, D. J. Roberts, W. H. Ouwehand, J. A. Todd, N. Soranzo, K. Suhre, D. S. Paul, C. S. Fox, R. M. Plenge, J. Danesh, H. Runz, A. S. Butterworth, Genomic atlas of the human plasma proteome Nature 558 (2018) 73-79.

[22]H. Shi, G. Kichaev, B. Pasaniuc, Contrasting the Genetic Architecture of 30 Complex Traits from Summary Association Data Am J Hum Genet 99 (2016) 139-153.

[23]B. Bulik-Sullivan, H. K. Finucane, V. Anttila, A. Gusev, F. R. Day, An atlas of genetic correlations across human diseases and traits 47 (2015) 1236-1241.

[24]A. C. Mcpherron, A.M. Lawler, S. J. Lee, Regulation of skeletal muscle mass in mice by a new TGFbeta superfamily member Nature (1997).

[25]N. F. Gonzalez-Cadavid, W. E. Taylor, K. Yarasheski,., I. Sinha-Hikim,., K. Ma,.., S. Ezzat,., R. Shen,., R. Lalani,., S. Asa,.. M. Mamita,. Organization of the human myostatin gene and expression in healthy men and HIV-infected men with muscle wasting Proc Natl Acad Sci U S A 95 (1998) 14938-14943.

[26]G. U. Zhiliang, D. Zhu, L. I. Ning, L. I. Hui, X. Deng, W. U. Changxin, The single nucleotide polymorphisms of the chicken myostatin gene are associated with skeletal muscle and adipose growth Science in China Ser C 47 (2004) 25-30.

[27]M. Schuelke, K. R. Wagner, L. E. Stolz, C. Hubner, T. Riebel, W. Komen, T. Braun, J. F. Tobin, S. J. Lee, Myostatin mutation associated with gross muscle hypertrophy in a child The New England journal of medicine 350 (2004) 2682-2688.

[28]S. Victoria, P. Leanne, S. M?Nica Senna, L. Nicholas, K. Ravi, S. Mridula, Prolonged absence of myostatin reduces sarcopenia Journal of Cellular Physiology 209 (2010) 866-873.

[29]T. A. White, N. K. Lebrasseur, Myostatin and Sarcopenia: Opportunities and Challenges - A Mini-Review Gerontology 60 (2014) 289-293. 
[30]R. H. Mak, P. Rotwein, Myostatin and insulin-like growth factors in uremic sarcopenia: the yin and yang in muscle mass regulation Kidney International 70 (2006) 410-412.

[31]D. A. Lowe, H. Degens, K. D. Chen, S. E. Alway, Glyceraldehyde-3-Phosphate Dehydrogenase Varies With Age in Glycolytic Muscles of Rats The Journals of Gerontology: Series A 55 (2000) B160-B164.

[32]A. H. Caswell, A.M. Corbett, Interaction of glyceraldehyde-3-phosphate dehydrogenase with isolated microsomal subfractions of skeletal muscle Journal of Biological Chemistry 260 (1985) 6892.

[33]S. M. Dagher, H. O. Hultin, Association of glyceraldehyde-3-phosphate dehydrogenase with the particulate fraction of chicken skeletal muscle Febs Journal 55 (2010) 185-192.

[34]R. D. Barber, D. W. Harmer, R. A. Coleman, B. J. Clark, GAPDH as a housekeeping gene: analysis of GAPDH mRNA expression in a panel of 72 human tissues Physiological genomics 21 (2005) 389-395.

[35]D. A. Lowe, H. Degens, K. D. Chen, S. E. Alway, Glyceraldehyde-3-Phosphate Dehydrogenase Varies With Age in Glycolytic Muscles of Rats J Gerontol A Biol Sci Med Sci 55 (2000) B160.

[36]0. Saitoh,., A. Fujisawa-Sehara,., Y. Nabeshima,.., M. Periasamy,. Expression of myogenic factors in denervated chicken breast muscle: isolation of the chicken Myf5 gene Nucleic Acids Research 21 (1993) 2503.

[37]S. Cresci,., L. D. Wright, J. A. Spratt, F. N. Briggs, D. P. Kelly, Activation of a novel metabolic gene regulatory pathway by chronic stimulation of skeletal muscle American Journal of Physiology 270 (1996) 1413-1420.

[38]R. W. Tsika, S. D. Hauschka, L. Gao,. M-creatine kinase gene expression in mechanically overloaded skeletal muscle of transgenic mice American Journal of Physiology 269 (1995) 665-674.

[39]J. J. Mccarthy, A.M. Fox, G. L. Tsika, L. Gao,., R. W. Tsika, beta-MHC transgene expression in suspended and mechanically overloaded/suspended soleus muscle of transgenic mice Am J Physiol 272 (1997) 1552-1561.

\section{Table 1}

Table 1. Genetic correlation analysis results between lean body mass and Blood plasma proteins

\section{Figures}




\begin{tabular}{lcl}
\multicolumn{1}{c}{ Chemical Name } & P value & Tissue \\
\hline selenomethylselenocysteine & 0.0088 & Blood \\
pirinixic acid & 0.0026 & Blood \\
methylselenic acid & 0.0032 & Blood \\
carbonyl sulfide & 0.0036 & Blood \\
chloramine-T & 0.0214 & Blood \\
perfluorodecanoic acid & 0.0010 & Blood \\
selenomethylselenocysteine & 0.0088 & Blood \\
pirinixic acid & 0.0026 & Blood \\
fusarenon-X & 0.0362 & Brain \\
apocarotenal & 0.0062 & Brain \\
decabromobiphenyl ether & 0.0002 & Brain \\
cadmium sulfide & 0.0282 & Brain \\
1-aminomethylphosphonic acid & 0.0198 & Brain \\
bafilomycin A1 & 0.0264 & Brain \\
cordycepin & 0.0224 & Brain \\
N-(4-methoxyphenyl)retinamide & 0.0408 & Brain \\
tobacco mosaic virus protein (103-112) & 0.0306 & Brain
\end{tabular}
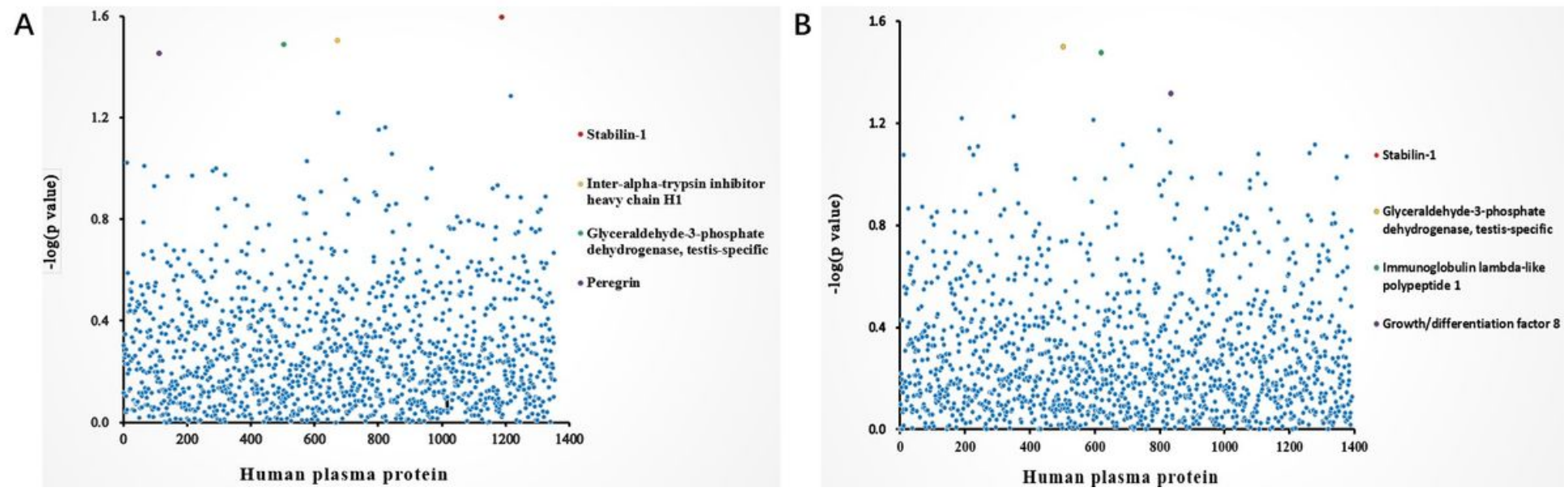

Figure 1

Plot of genetic correlation analysis results between plasma proteins and lean body mass (A, appendicular lean body mass; $\mathrm{B}$, whole lean body mass) 